TRANSACTIONS OF THE

AMERICAN MATHEMATICAL SOCIETY

Volume 360, Number 12, December 2008, Pages 6545-6554

S 0002-9947(08)04473-5

Article electronically published on June 26, 2008

\title{
A PROBABILISTIC APPROACH TO BOUNDED/POSITIVE SOLUTIONS FOR SCHRÖDINGER OPERATORS WITH CERTAIN CLASSES OF POTENTIALS
}

\author{
ROSS G. PINSKY
}

Abstract. Consider the equation

$$
\frac{1}{2} \Delta u-V u=0 \text { in } R^{d},
$$

for $d \geq 3$. For certain classes of potentials $V$, we use probabilistic tools to study the bounded solutions and the positive solutions for $\left(^{*}\right)$. A primary motivation is to offer probabilistic intuition for the results.

\section{INTRODUCTION AND STATEMENT OF RESULTS}

In this paper we use probabilistic tools to study the bounded solutions and the positive solutions for Schrödinger operators with certain classes of potentials in $R^{d}$. We restrict to $d \geq 3$ because the questions we ask have trivial answers for $d=1,2$. A primary motivation here is to offer probabilistic intuition for the results.

We consider classical solutions to the equation

$$
\frac{1}{2} \Delta u-V u=0 \text { in } R^{d}
$$

where $V$ is Hölder continuous. As usual, we let $V^{+}=V \vee 0$ and $V^{-}=-(V \wedge 0)$, so that $V=V^{+}-V^{-}$. To avoid trivialities, we assume that $V \not \equiv 0$. Let $X(t)$ be a $d$-dimensional Brownian motion. When the Brownian motion starts from $x \in R^{d}$, we denote probabilities by $P_{x}$ and the corresponding expectations by $E_{x}$.

Recall that the Green's function for $\frac{1}{2} \Delta$ in $R^{d}, d \geq 3$, is given by $G(x, y)=$ $\frac{2}{(d-2) \omega_{d}}|y-x|^{2-d}$, where $\omega_{d}$ is the surface measure of the unit sphere in $R^{d}$. As is well known [3], for any nonnegative function $\phi$, one has

$$
E_{x} \int_{0}^{\infty} \phi(X(t)) d t=\int_{R^{d}} G(x, y) \phi(y) d y=\frac{2}{(d-2) \omega_{d}} \int_{R^{d}} \frac{\phi(y)}{|y-x|^{d-2}} d y .
$$

The following assumption will always be made on the negative part $V^{-}$of the potential $V$ :

\section{Assumption 1.}

$$
\sup _{x \in R^{d}} E_{x} \int_{0}^{\infty} V^{-}(X(t)) d t=\frac{2}{(d-2) \omega_{d}} \sup _{x \in R^{d}} \int_{R^{d}} \frac{V^{-}(y)}{|y-x|^{d-2}} d y<1 .
$$

Received by the editors June 26, 2006 and, in revised form, January 16, 2007.

2000 Mathematics Subject Classification. Primary 60H30, 35J10.

Key words and phrases. Liouville theorem, bounded solutions, positive solutions, Schrödinger equation.

(C)2008 American Mathematical Society Reverts to public domain 28 years from publication 
By Khasminskii's lemma [14, Lemma B.1.2], Assumption 1 guarantees that

$$
\sup _{x \in R^{d}} E_{x} \exp \left(\int_{0}^{\infty} V^{-}(X(t)) d t\right)<\infty .
$$

Remark. Actually it is (1.3) that we will need throughout the paper, not Assumption 1 However, it seems more appropriate to state the results with a purely analytic condition.

The condition (1.3) is more than sufficient to guarantee that the operator $\frac{1}{2} \Delta-V$ is subcritical; that is, it possesses a positive Green's function. In particular, then the cone $C_{V} \equiv\left\{u: \frac{1}{2} \Delta u-V u=0\right.$ and $u>0$ in $\left.R^{d}\right\}$ of positive solutions for the operator $\frac{1}{2} \Delta-V$ in $R^{d}$ is nonempty (and nontrivial since positive constants are not solutions) 13. In order not to disrupt the exposition, we defer an explanation of this until the end of this section. The Liouville property is said to hold for the potential $V$ if the only bounded solution of (1.1) is $u \equiv 0$.

Now consider the positive part of the potential $V$. One has simultaneously for all $x$ either $P_{x}\left(\int_{0}^{\infty} V^{+}(X(t)) d t=\infty\right)=0$ or 1 , since the event $\left\{\int_{0}^{\infty} V^{+}(X(t)) d t=\infty\right\}$ belongs to the tail $\sigma$-algebra of Brownian motion, and this $\sigma$-algebra is trivial. Note that by (1.2) and Assumption 1, we have a fortiori that $P_{x}\left(\int_{0}^{\infty} V^{-}(X(t)) d t=\infty\right)=$ 0 . Our first result gives, among other things, a probabilistic characterization of the Liouville property.

Theorem 1. Let $V$ be Hölder continuous and let $V^{-}$satisfy Assumption 1, Let $X(t)$ be a d-dimensional Brownian motion. Then the Liouville property holds (that is, there are no bounded solutions to (1.1)) if and only if

$$
\int_{0}^{\infty} V^{+}(X(t)) d t=\infty, \text { a.s. }
$$

In the case that the Liouville property fails, let

$$
u_{0}(x)=E_{x} \exp \left(-\int_{0}^{\infty} V(X(t)) d t\right) .
$$

Then $u_{0}$ is a bounded, positive solution to (1.1). Every bounded solution to (1.1) is a multiple of $u_{0}$. In particular, there are no bounded, sign-changing solutions.

Remark 1 . In the case that the potential $V$ is nonnegative, the condition

$$
\int_{0}^{\infty} V(X(t)) d t=\infty
$$

can easily be shown to be equivalent to the strong stability on $L^{1}\left(R^{d}\right)$ of the semigroup $T_{t}$ corresponding to the operator $\frac{1}{2} \Delta-V$; that is, to the condition $\lim _{t \rightarrow \infty}\left\|T_{t} f\right\|_{1}=0$, for all $f \in L^{1}\left(R^{d}\right)$. This connection is developed probabilistically in 2 .

Remark 2. In light of Theorem 1 and (1.2), it follows that $\int_{R^{d}} \frac{V^{+}(x)}{\left.x\right|^{d-2}} d x=\infty$ is a necessary condition for the Liouville property to hold. In the case that $V \geq 0$, an alternative purely analytic proof of this necessary condition for the Liouville property can be found in [6]. See also [7] which treats the Liouville problem from a potential theoretic point of view in the case that the potential is an appropriate nonnegative measure and the space $R^{d}$ is replaced by a Riemannian manifold. Yehuda Pinchover has pointed out to me that Lemma 3.3 and Proposition 3.4 in 
12 can be used to give a completely analytic proof that the Liouville property fails if $V^{-}$satisfies Assumption 1 and $V^{+}$satisfies $\int_{R^{d}} \frac{V^{+}(x)}{|x|^{d-2}} d x<\infty$.

In certain cases, the condition $\int_{R^{d}} \frac{V^{+}(x)}{|x|^{d-2}} d x=\infty$ is not only necessary but also sufficient in order that $\int_{0}^{\infty} V^{+}(X(t)) d t=\infty$ a.s., or equivalently by Theorem 1 , in order that the Liouville property hold. In particular, this is true in the case that $V^{+}$is radially symmetric.

Proposition 1. Let $\phi \geq 0$ be radially symmetric. One has

$$
\int_{0}^{\infty} \phi(X(t)) d t=\infty \text { a.s. if and only if } \int_{R^{d}} \frac{\phi(x)}{|x|^{d-2}} d x=\infty .
$$

Thus, if $V^{-}$satisfies Assumption 1 and $V^{+}$is radially symmetric, then by Theorem 1. the Liouville property holds if and only if $\int_{R^{d}} \frac{V^{+}(x)}{|x|^{d-2}} d x=\infty$.

Remark. In the case that $V$ is nonnegative and radially symmetric, Brezis, Chipot and Xie 4] have recently given an elementary analytic proof that the Liouville property holds if and only if $\int_{R^{d}} \frac{V(x)}{|x|^{d-2}} d x=\infty$. Alternative proofs of (1.4) can be found in [2, Corollary 3.11] and [1, Theorem 3.7] in the context of strong stability in $L^{1}\left(R^{d}\right)$ of the semigroup corresponding to the operator $\frac{1}{2} \Delta-V$.

Another case besides the radial one where the condition $\int_{R^{d}} \frac{V^{+}(x)}{|x|^{d-2}} d x=\infty$ is necessary and sufficient in order that $\int_{0}^{\infty} V^{+}(X(t)) d t=\infty$ a.s. is the case that $V^{+}$ decays at least quadratically. Indeed, the following result follows from [3].

Theorem BA-P. (i) Assume that $0 \leq \phi(x) \leq \frac{C}{(1+|x|)^{2}}$ for some $C>0$. Then $\int_{0}^{\infty} \phi(X(t)) d t<\infty$ a.s. if and only if $\int_{R^{d}} \frac{\phi(x)}{|x|^{d-2}} d x<\infty$.

(ii) Let $\psi$ be any positive, nondecreasing function on $[0, \infty)$ satisfying $\lim _{r \rightarrow \infty} \psi(r)=\infty$. Then there exists a function $\phi$ satisfying $0 \lessgtr \phi(x) \leq \frac{\psi(|x|)}{(1+|x|)^{2}}$ for which $\int_{0}^{\infty} \phi(X(t)) d t<\infty$ a.s. but $\int_{R^{d}} \frac{\phi(x)}{|x|^{d-2}} d x=\infty$.

In order not to disrupt the exposition, we wait until the end of this section to explain how Theorem BA-P follows from [3].

Theorem BA-P(i) and Theorem 1 give the following immediate corollary.

Corollary 1. If $V^{-}$satisfies Assumption 1 and $V^{+}(x) \leq \frac{C}{(1+|x|)^{2}}$ for some $C>0$, then the Liouville property holds if and only if $\int_{R^{d}} \frac{V^{+}(x)}{|x|^{d-2}} d x=\infty$.

Remark 1. Theorem 1 and Theorem BA-P(ii) show that the quadratic decay condition on $V^{+}$is sharp with regard to $\int_{R^{d}} \frac{V^{+}(x)}{|x|^{d-2}} d x<\infty$ being the necessary and sufficient condition for the Liouville condition to hold.

Remark 2. It follows immediately from Theorem 1 and Theorem BA-P that a sufficient condition for the Liouville property to hold is that

$$
\int_{R^{d}}\left(V^{+}(x) \wedge \frac{1}{(1+|x|)^{2}}\right) \frac{1}{|x|^{d-2}} d x=\infty .
$$

It is known that if the potential $V$ decays at least quadratically, then the cone $C_{V}$ of positive solutions for $\frac{1}{2} \Delta-V$ is one-dimensional [13, Chapter 8.3]. Thus, if $V$ decays at least quadratically and $V^{-}$satisfies Assumption 1 , then by Corollary 1 
the unique positive solution (up to positive multiples) will be bounded if and only if $\int_{R^{d}} \frac{V^{+}(x)}{|x|^{d-2}} d x<\infty$.

We now give another "smallness" condition on the potential $V$ which guarantees that the cone $C_{V}$ is one-dimensional. Theorem 1 showed that if $V^{-}$satisfies Assumption 1 and $\int_{0}^{\infty} V^{+}(X(s)) d s<\infty$ a.s., then there exists a unique bounded, positive solution to (1.1) up to positive multiples. Also, we noted that a sufficient condition for this integral to be finite is that $\int_{R^{d}} \frac{V^{+}(x)}{|x|^{d-2}} d x<\infty$. Using a mixture of probabilistic and analytic techniques, we will show that if this last integral condition on $V^{+}$is strengthened a bit, and if we assume that $V^{-}=0$, then all positive solutions must bounded, from which it then follows that the cone $C_{V}$ of positive solutions is one-dimensional.

\section{Assumption 2.}

$$
\sup _{x \in R^{d}} \int_{R^{d}} \frac{V^{+}(y)}{|y-x|^{d-2}} d y<\infty .
$$

Theorem 2. Let $V^{+}$satisfy Assumption 2 and let $V^{-}=0$. Then the cone $C_{V}$ of positive solutions to (1.1) is one-dimensional. It is generated by the bounded function $u_{0}$ appearing in Theorem 1 .

Remark. In the literature, a potential $V$ satisfying Assumption 2 with $V^{+}$replaced by $|V|$ is called an $H$-bounded perturbation of $\frac{1}{2} \Delta$. (Actually, in the present context, the definition of an $H$-bounded perturbation is equivalent to the definition of a $G$-bounded perturbation.) For more on these kinds of perturbations, see for example [12, section 2]. The result in Theorem 2 doesn't seem to be in the literature. However, Yehuda Pinchover has pointed out to me that a purely analytic proof can be made by combining a number of results of his in [12] and 11. We are inclined to believe that the condition $\int_{R^{d}} \frac{V^{+}(y)}{|y-x|^{d-2}} d y<\infty$, for all $x$ (which is of course equivalent to the condition $\int_{R^{d}} \frac{V^{+}(y)}{\mid y^{d-2}} d y<\infty$, since $V$ is locally bounded), is insufficient to guarantee that the cone $C_{V}$ is one-dimensional. Note that by Theorem 11, it follows that if $V^{+}$satisfies $\int_{R^{d}} \frac{V^{+}(y)}{|y|^{d-2}} d y<\infty$ and $V^{-}$satisfies Assumption 1. then the cone $C_{V}$ will be one-dimensional if and only if there are no unbounded, positive solutions to (1.1).

In the case that $V^{+}$is radially symmetric, Theorem 2 does hold with Assumption 2 replaced by the condition $\int_{R^{d}} \frac{V^{+}(x)}{\mid x x^{d-2}} d x<\infty$, which by Proposition 1, is the necessary and sufficient condition for the Liouville property to hold. As the proof below will show, the reason for this is that in the radial case, the supremum in (1.5) occurs when $x=0$.

Corollary 2. Let $V^{+}$be radially symmetric and let $V^{-}$satisfy Assumption 1 , If $\int_{R^{d}} \frac{V^{+}(x)}{|x|^{d-2}} d x<\infty$, then the cone $C_{V}$ of positive solutions to (1.1) is onedimensional and contains only bounded, functions; namely the positive multiples of the bounded function $u_{0}$ appearing in Theorem 1 . If $V^{-}$is also radially symmetric, then this function $u_{0}$ is radially symmetric.

Remark. In the case that $V$ is radially symmetric and $\int_{R^{d}} \frac{V(x)}{|x|^{d-2}} d x=\infty$, the cone $C_{V}$ of positive solutions is not necessarily one-dimensional, nor are all solutions necessarily radially symmetric. For example, if $V \equiv 1$, then for each $v \in R^{d}$ 
satisfying $|v|=\sqrt{2}$, the function $\exp (v \cdot x)$ is a solution to (1.1). On the other hand, the condition $\int_{R^{d}} \frac{V(x)}{|x|^{d-2}} d x=\infty$ does not rule out quadratic decay, and under such a decay rate the cone $C_{V}$ is one-dimensional, as noted above. For a detailed analysis of the structure of the positive solutions in the radial case, see [10].

Proof of Corollary 2, Let $\tau_{r}=\inf \{t \geq 0:|X(t)|=r\}$. Then by the strong Markov property and the radial symmetry of $V^{+}$, we have for $x \in R^{d}$,

$$
\begin{aligned}
& E_{0} \int_{0}^{\infty} V^{+}(X(t)) d t=E_{0}\left(\int_{0}^{\tau_{|x|}} V^{+}(X(t)) d t+\int_{\tau_{|x|}}^{\infty} V^{+}(X(t)) d t\right) \\
& \geq E_{x} \int_{0}^{\infty} V^{+}(X(t)) d t
\end{aligned}
$$

which in conjunction with (1.2) shows that

$$
\sup _{x \in R^{d}} \int_{R^{d}} \frac{V^{+}(y)}{|y-x|^{d-2}} d y=\int_{R^{d}} \frac{V^{+}(y)}{|y|^{d-2}} d y .
$$

We now give some of the proof of Theorem 2 in order to emphasize the probabilistic intuition for condition (1.5). The missing details will be completed in Section 3. By Theorem 1 and (1.2), in order to prove the theorem it is enough to show that there are no unbounded, positive solutions.

Assume to the contrary that $u$ is an unbounded, positive solution. By the Feynman-Kac formula and Assumption 1, $u$ satisfies

$$
u(x)=E_{x} \exp \left(-\int_{0}^{\tau_{n}} V^{+}(X(s)) d s\right) u\left(X\left(\tau_{n}\right)\right),
$$

for $|x| \leq n$, where $\tau_{n}=\inf \{t \geq 0:|X(t)|=n\}$. Let $w_{n}(x)=E_{x} u\left(X\left(\tau_{n}\right)\right)$, for $x \in \bar{B}_{n}$. Then $w_{n}$ is harmonic in $B_{n}$ and by (1.7),

$$
u(x) \leq w_{n}(x) .
$$

By a standard compactness argument and the Harnack inequality, either $\left\{w_{n}\right\}$ has a convergent subsequence which converges to a limiting positive harmonic function in all of $R^{d}$, or else $\lim _{n \rightarrow \infty} w_{n}(x)=\infty$, for all $x \in R^{d}$. In light of (1.8) and the assumed unboundedness of $u$, the first possibility would lead to an unbounded, positive harmonic function in all of $R^{d}$. But this is impossible because the only positive harmonic functions are the constants. Thus, we conclude that

$$
\lim _{n \rightarrow \infty} E_{x} u\left(X\left(\tau_{n}\right)\right)=\infty \text { for all } x .
$$

Using conditional expectation, we have from (1.7) that

$$
\begin{aligned}
& u(0)=E_{0} \exp \left(-\int_{0}^{\tau_{n}} V^{+}(X(s)) d s\right) u\left(X\left(\tau_{n}\right)\right) \\
& =\int_{\partial B_{n}} E_{0}\left(\exp \left(-\int_{0}^{\tau_{n}} V^{+}(X(s)) d s\right) \mid X\left(\tau_{n}\right)=y\right) u(y) \sigma(d y) \text { for all } n,
\end{aligned}
$$

where $\sigma(d y)$ is normalized surface measure on $\partial B_{n}$. From (1.9) with $x=0$, we have

$$
\lim _{n \rightarrow \infty} \int_{\partial B_{n}} u(y) \sigma(d y)=\infty .
$$


If we knew that for some $M$

$$
\lim _{n \rightarrow \infty} \inf _{y \in \partial B_{n}} P_{0}\left(\int_{0}^{\tau_{n}} V^{+}(X(s)) d s \leq M \mid X\left(\tau_{n}\right)=y\right)>0,
$$

then it would follow from (1.11) that

$$
\lim _{n \rightarrow \infty} \int_{\partial B_{n}} E_{0}\left(\exp \left(-\int_{0}^{\tau_{n}} V^{+}(X(s)) d s\right) \mid X\left(\tau_{n}\right)=y\right) u(y) \sigma(d y)=\infty,
$$

which would contradict (1.10).

Now a sufficient condition to guarantee (1.12) is that the collection of distributions

$$
\left\{\int_{0}^{\tau_{n}} V^{+}(X(s)) d s \text { under } P_{0}\left(\cdot \mid X\left(\tau_{n}\right)=y\right): y \in \partial B_{n}, n=1,2, \cdots\right\}
$$

be tight. Also, a sufficient condition for this tightness is the uniform boundedness of the expectations:

$$
\sup _{n} \sup _{y \in \partial B_{n}} E_{0}\left(\int_{0}^{\tau_{n}} V^{+}(X(s)) d s \mid X\left(\tau_{n}\right)=y\right)<\infty .
$$

Now we can write an explicit analytic expression for the expectation in (1.13), using the Green's function and the Poisson kernel. In Section 3 we complete the proof of Theorem 2 by showing that condition (1.5) is sufficient for (1.13) to hold. Note that condition (1.5) has a probabilistic interpretation in the spirit of (1.13) since

$$
\sup _{x \in R^{d}} E_{x} \int_{0}^{\infty} V^{+}(X(s)) d s=\sup _{x \in R^{d}} \int_{R^{d}} \frac{V^{+}(y)}{|y-x|^{d-2}} d y .
$$

We now return to the probabilistic condition on $V^{+}$which was shown in Theorem 1 to be equivalent to the Liouville condition: $\int_{0}^{\infty} V^{+}(X(t)) d t=\infty$. It is probably not possible to find a concrete analytic condition on $V^{+}$which characterizes in general whether or not $\int_{0}^{\infty} V^{+}(X(t)) d t=\infty$. (For an abstract, potential theoretic condition which characterizes this, see [2].) A more tractable task is to identify interesting classes of nonnegative potentials $V$, besides those nonnegative potentials which are radially symmetric or which decay at least quadratically, for which the condition $\int_{R^{d}} \frac{V(x)}{|x|^{d-2}} d x=\infty$ characterizes the Liouville property. The case in which $V$ is supported in a countable collection of disjoint balls with appropriate side conditions will be treated elsewhere in work by a student $[8$, by adapting the method used recently in [5], where an interesting related question was investigated. Let $B(c, r)$ denote the open ball of radius $r$ centered at $c \in R^{d}$. Consider a countable collection of disjoint, closed balls $\left\{\bar{B}\left(c_{n}, r_{n}\right)\right\}_{n=1}^{\infty}$ in $R^{d}, d \geq 3$. The problem considered in [5] is to characterize those collections of balls for which the probability of never hitting $\bigcup_{n=1}^{\infty} \bar{B}\left(c_{n}, r_{n}\right)$ is positive; or equivalently, to characterize those collections of balls for which there exists a nonconstant, bounded harmonic function $u$ in $R^{d}-\bigcup_{n=1}^{\infty} \bar{B}\left(c_{n}, r_{n}\right)$ satisfying $u=1$ on $\bigcup_{n=1}^{\infty} \partial B\left(c_{n}, r_{n}\right)$. This problem can be posed as a "singular" Liouville-type problem as follows.

Let $A=\bigcup_{n=1}^{\infty} \bar{B}\left(c_{n}, r_{n}\right)$ and let $V=0$ on $R^{d}-A$ and $V=\infty$ on $A$. In an appropriate sense, the function $u=1_{R^{d}-A}$ solves $\frac{1}{2} \Delta u-V u=0$. We call this solution the trivial one, and say that the Liouville property holds if there are no nontrivial, bounded solutions. Then the Liouville property holds if and only if the probability of avoiding $\cup_{n=1}^{\infty} \bar{B}\left(c_{n}, r_{n}\right)$ forever is zero. In [5] it was shown that if all the balls are $\epsilon$ separated and if $\sup _{n \geq 1} r_{n}^{d-2}\left|c_{n}\right|^{2}<\infty$, then a necessary and 
sufficient condition for there to be a positive probability of avoiding $\cup_{n=1}^{\infty} \bar{B}\left(c_{n}, r_{n}\right)$ forever is that $\sum_{n=1}^{\infty}\left(\frac{r_{n}}{\left|c_{n}\right|}\right)^{d-2}<\infty$. Without any conditions on the balls, the finiteness of the above sum is sufficient for the balls to be avoidable.

The authors also show that the condition $\sup _{n \geq 1} r_{n}^{d-2}\left|c_{n}\right|^{2}<\infty$ is sharp with regard to the convergence of the above sum being the necessary and sufficient condition. We point out that it is possible to have a situation where the balls are unavoidable, but the total time spent in them is almost surely finite. Indeed, if $r_{n}$ is say, bounded, and $\sum_{n=1}^{\infty} \frac{r_{n}^{d}}{\left|c_{n}\right|^{d-2}}<\infty$, then $E_{0} \int_{0}^{\infty} 1_{\cup_{n=1}^{\infty} \bar{B}\left(c_{n}, r_{n}\right)}(X(t)) d t=$ $\frac{1}{(d-2) \omega_{d}} \sum_{n=1}^{\infty} \int_{B\left(c_{n}, r_{n}\right)} \frac{1}{|x|^{d-2}} d x<\infty$. Thus, for example, choosing $r_{n}$ and $c_{n}$ such that $r_{n}^{d-2}\left|c_{n}\right|^{2}=1$ and $\left|c_{n}\right|=n^{\frac{1}{d}}$, then $\sum_{n=1}^{\infty}\left(\frac{r_{n}}{\left|c_{n}\right|}\right)^{d-2}=\infty$ but $\sum_{n=1}^{\infty} \frac{r_{n}^{d}}{\left|c_{n}\right|^{d-2}}<\infty$.

We now return to explain how Theorem BA-P follows from [3].

Proof of Theorem BA-P. From [3, Theorems 1 and 2 and Corollary 4], it follows that if $\phi$ satisfies the bounds in part (i), then the $P_{x}$-almost sure infiniteness/finiteness of $\int_{0}^{\infty} \phi\left(X(t) d t\right.$ is equivalent to the infiniteness/finiteness of $E_{x} \int_{0}^{\infty} \phi(X(t) d t$. Part (i) now follows from (1.2). (In Corollary 4 in [3], there is a typographical error. The term $b(y)$ there should actually be $b^{2}(y)$. The term $b^{2}$ which is supposed to appear in the integral there plays the role of $\phi$ in (1.2). Also, in the proof of Theorem 2 in 3, the limit appearing in the displayed formula between (3.3) and (3.4) should be outside of the expectation, and the statement in (3.4) should be in probability rather than almost surely. Then (3.7) there will hold for a sequence of times converging to infinity rather than for a continuum of times.)

It was also shown in [3] that for each function $\psi$ as in part (ii) of the present theorem, it is possible to construct a function $\phi$ which satisfies the growth condition in part (ii) and is such that $\int_{0}^{\infty} \phi(X(t)) d t<\infty$ a.s. $\left[P_{x}\right]$, but $E_{x} \int_{0}^{\infty} \phi(X(t)) d t=$ $\infty$. (See Theorem 3 in [3], in conjunction with Theorems 1 and 2 and Corollary 4 there. The role of $\phi$ here is played by $|\hat{b}|^{2}$ in the proof of Theorem 3 in [3].) Thus, part (ii) now also follows from (1.2).

We end this section with the explanation for the claim that condition (1.3) is more than sufficient to guarantee that $\frac{1}{2} \Delta-V$ is a subcritical operator. In fact, it is enough to assume that $E_{x} \exp \left(\int_{0}^{\infty} V^{-}(X(t)) d t\right)<\infty$, for all $x$ (or equivalently, for some $x)$. By standard Schauder estimates, $u(x) \equiv E_{x} \exp \left(\int_{0}^{\infty} V^{-}(X(t)) d t\right)$ is a positive solution for the operator $\frac{1}{2} \Delta+V^{-}$. Since $u \geq 1$, it follows that $\hat{u} \equiv u-\frac{1}{2}$ is a positive super-solution for $\frac{1}{2} \Delta-V$, but not a solution; that is, $\frac{1}{2} \Delta u-V u \lessgtr 0$. But this is a sufficient condition for the subcriticality of $\frac{1}{2} \Delta-V$ [13, Theorem 4.3.9].

We prove Theorem 1 and Proposition 1 in Section 2 and complete the proof of Theorem 2 in Section 3.

\section{Proofs of Theorem 1 and Proposition 1}

Proof of Theorem 1. First assume that $\int_{0}^{\infty} V^{+}(X(t)) d t=\infty$ a.s. Let $u$ be a bounded solution to (1.1). By the Feynman-Kac formula and Assumption 1, we have

$$
u(x)=E_{x} u(X(t)) \exp \left(-\int_{0}^{t} V(X(s)) d s\right) \text { for all } t>0
$$


Now let $t \rightarrow \infty$. By Assumption [1, the dominated convergence theorem can be applied to give $u \equiv 0$.

Conversely, assume that $\int_{0}^{\infty} V^{+}(X(t)) d t<\infty$ a.s. By Assumption 1, the function $u_{0}$ defined in the statement of part (i) of the theorem is bounded. By standard Schauder estimates and the Feynman-Kac formula, the function $u_{0}$ will be a solution to (1.1).

It remains to show that every bounded solution is a multiple of $u_{0}$. Let $u$ be any bounded solution. Let $x \in R^{d}$. By the Feynman-Kac formula and Assumption 1. $u(X(t)) \exp \left(-\int_{0}^{t} V(X(s)) d s\right)$ is a $P_{x^{x}}$-martingale. Since this martingale is positive, it converges $\left[P_{x}\right]$ almost surely, and since it is dominated by $M \exp \left(\int_{0}^{\infty} V^{-}(X(t)) d t\right)$, where $M=\sup _{x \in R^{d}}|u(x)|$, it follows from Assumption 1 and the dominated convergence theorem that the convergence is also in $L_{1}$. By Assumption 1 and by the assumption on $V^{+}$, it follows that $\int_{0}^{t} V(X(s)) d s$ converges $\left[P_{x}\right]$ almost surely to a finite limit. Thus, we conclude that $U \equiv \lim _{t \rightarrow \infty} u(X(t))$ exists $P_{x}$ almost surely and that

$$
u(x)=E_{x} U \exp \left(-\int_{0}^{\infty} V(X(t)) d t\right) .
$$

Since $U$ is measurable with respect to the tail $\sigma$-algebra of the Brownian motion, and since this $\sigma$-algebra is trivial, it follows that $U \equiv c_{x}$ a.s. $\left[P_{x}\right]$, for some constant $c_{x}$. The limit $c_{x}$ is in fact independent of $x$. Indeed, let $x_{1}, x_{2} \in R^{d}$ and let $R>\left|x_{1}\right|,\left|x_{2}\right|$. By the strong Markov property and the fact that $U$ is a tail event, it follows that

$$
P_{x_{i}}(U \in \cdot)=E_{x_{i}} P_{X\left(\tau_{R}\right)}(U \in \cdot) .
$$

As is well-known, the harmonic measure on $\partial B_{R} \equiv\{|x|=R\}$ is mutually absolutely continuous with respect to Lebesgue measure; that is, the distribution of $X\left(\tau_{R}\right)$ under $P_{x_{i}}$ is mutually absolutely continuous with Lebesgue measure. Since $\lim _{t \rightarrow \infty} u(X(t))=c_{x_{i}}$ a.s. $\left[P_{x_{i}}\right]$, it follows from (2.3) and the above-mentioned absolute continuity that $c_{y}=c_{x_{i}}$ for Lebesgue-almost all $y \in \partial B_{R}$. Since this is true for $i=1,2$, we conclude that $c_{x_{1}}=c_{x_{2}}$. Calling the common value of this constant $c$, it follows from (2.2) that $u=c u_{0}$.

Proof of Proposition 1. The theorem will follow from (1.2) if we show that the condition $\int_{0}^{\infty} V^{+}(X(t)) d t<\infty$ a.s. guarantees that $E_{x} \int_{0}^{\infty} V^{+}(X(t)) d t<\infty$. Assume that $\int_{0}^{\infty} V(X(t)) d t<\infty$ a.s. By Theorem 1, $u_{0}(x) \equiv E_{x} \exp \left(-\int_{0}^{\infty} V(X(t)) d t\right)$ is a bounded solution to (1.1). By the strong Markov property and the radial symmetry of $V^{+}$, the distribution of $\int_{0}^{\infty} V^{+}(X(t)) d t$ under $P_{x}$ is the same as the distribution of $\int_{\tau_{|x|}}^{\infty} V^{+}(X(t)) d t$ under $P_{0}$. Thus, this distribution is stochastically decreasing in $|x|$. From this and the stochastic representation of $u_{0}$ above, it follows that $u_{0}$ is bounded from 0 . From the fact that $\frac{1}{2} \Delta u_{0}=V u_{0}$, it follows that $u_{0}(X(t))-\int_{0}^{t}\left(V u_{0}\right)(X(s)) d s$ is a local martingale. Thus,

$$
E_{x} u_{0}\left(X\left(\tau_{n}\right)\right)=u_{0}(0)+E_{x} \int_{0}^{\tau_{n}}\left(V u_{0}\right)(X(s)) d s .
$$

By Assumption 1 and the boundedness of $u_{0}, E_{x} \int_{0}^{\tau_{n}}\left(V^{-} u_{0}\right)(X(s)) d s$ is bounded independent of $n$. Thus, letting $n \rightarrow \infty$ in (2.4), and using the boundedness from 0 of $u_{0}$, we conclude that $E_{x} \int_{0}^{\infty} V^{+}(X(t)) d t<\infty$. 


\section{Completion of the Proof of Theorem 2}

We began the proof in the first section of the paper. To complete the proof, it remains to show that if (1.5) holds, then so does (1.13).

Let $P^{n}(x, y)$ denote the Poisson kernel for $\frac{1}{2} \Delta$ in the ball $B_{n}=\left\{x \in R^{d}:|x|<\right.$ $n\}$. That is, for each $y \in \partial B_{n}, P^{n}(\cdot, y)$ is harmonic in $B_{n}$, satisfies $\lim _{x \rightarrow z} P^{n}(x, y)$ $=0$ for $y \neq z \in \partial B_{n}$, and $\lim _{x \rightarrow y} P^{n}(x, y)=\infty$. One has [9]

$$
P^{n}(x, y)=\frac{1}{n \omega_{d}} \frac{n^{2}-|x|^{2}}{|y-x|^{d}} .
$$

Let $G^{n}(x, z)$ denote the Green's function for the Brownian motion killed upon exiting $B_{n}$. (That is, $G^{n}$ is the Green's function for $\frac{1}{2} \Delta$ in $B_{n}$ with the Dirichlet boundary condition.) One has 9 ]

$$
G^{(n)}(x, z)=\frac{2}{(d-2) \omega_{d}}\left(|z-x|^{2-d}-\left(\frac{|z|}{n}\right)^{2-d}\left|\frac{n^{2}}{|z|^{2}} z-x\right|^{2-d}\right) .
$$

Brownian motion in $B_{n}$, conditioned to exit at $y \in \partial B_{n}$, is generated by an $h$ transform of the original generator, with $h(x)=P^{n}(x, y)$ [13, chapter 7 , section $2]$. Let $G^{n, y}(x, z)$ denote the Green's function for the Brownian motion killed upon exiting $B_{n}$ and conditioned to exit at $y \in \partial B_{n}$. Then 13 , chapter 4 , sections 1 and $2]$

$$
G^{n, y}(x, z)=\frac{G^{n}(x, z) P^{n}(z, y)}{P^{n}(x, y)} .
$$

Using the above formulas along with (1.5), we can prove (1.13). Note that in the case $x=0$, (3.2) simplifies to $G^{n}(0, z)=\frac{2}{(d-2) \omega_{d}}\left(|z|^{2-d}-n^{2-d}\right)=\frac{2}{(d-2) \omega_{d}} \frac{n^{d-2}-|z|^{d-2}}{|z|^{d-2} n^{d-2}}$. Letting $C=\frac{2}{(d-2) \omega_{d}}$, we have

$$
\begin{aligned}
& E_{0}\left(\int_{0}^{\tau_{n}} V^{+}(X(s)) d s \mid X\left(\tau_{n}\right)=y\right)=\int_{B_{n}} G^{n, y}(0, z) V^{+}(z) d z \\
& =\int_{B_{n}} G^{n}(0, z) \frac{P^{n}(z, y)}{P^{n}(0, y)} V^{+}(z) d z=C \int_{B_{n}} \frac{n^{d-2}-|z|^{d-2}}{|z|^{d-2} n^{d-2}} \frac{n^{2}-|z|^{2}}{|z-y|^{d}} \frac{|y|^{d}}{n^{2}} V^{+}(z) d z \\
& =C \int_{B_{n}} \frac{n^{d-2}-|z|^{d-2}}{|z|^{d-2}} \frac{n^{2}-|z|^{2}}{|z-y|^{d}} V^{+}(z) d z \\
& =C \int_{B_{\frac{n}{2}}}\left(n^{d-2}-|z|^{d-2}\right) \frac{n^{2}-|z|^{2}}{|z-y|^{d}} \frac{V^{+}(z)}{|z|^{d-2}} d z \\
& +C \int_{B_{n}-B_{\frac{n}{2}}} \frac{n^{d-2}-|z|^{d-2}}{|z|^{d-2}} \frac{n^{2}-|z|^{2}}{|z-y|^{2}} \frac{V^{+}(z)}{|z-y|^{d-2}} d z .
\end{aligned}
$$

For $|z|<\frac{n}{2}$, it is clear that $\left(n^{d-2}-|z|^{d-2}\right) \frac{n^{2}-|z|^{2}}{|z-y|^{d}}$ is bounded independent of $y \in \partial B_{n}$ and $n$, and by (1.5), we have of course $\int_{B_{n}} \frac{V^{+}(z)}{|z|^{d-2}} d z \leq \int_{R^{d}} \frac{V^{+}(z)}{|z|^{d-2}} d z<\infty$. Thus, the integral over $B_{\frac{n}{2}}$ on the right hand side of (3.4) is bounded independent of $y \in \partial B_{n}$ and $n$.

Now consider the integral over $B_{n}-B_{\frac{n}{2}}$. Using the fact that $n^{d-2}-|z|^{d-2} \leq$ $c_{1}(n-|z|) n^{d-3}$, for some $c_{1}>0$, and that $|z-y| \geq n-|z|$, it follows that for 
$\frac{n}{2} \leq|z|<n$

$$
\frac{n^{d-2}-|z|^{d-2}}{|z|^{d-2}} \frac{n^{2}-|z|^{2}}{|z-y|^{2}} \leq c_{2} \frac{(n-|z|) n^{d-3}}{n^{d-2}} \frac{(n-|z|) n}{(n-|z|)^{2}}=c_{2}
$$

for some $c_{2}>0$. Using this with (1.5) now shows that the integral over $B_{n}-B_{\frac{n}{2}}$ on the right hand side of (3.4) is also bounded independent of $y \in \partial B_{n}$ and $n$. This completes the proof of the theorem.

\section{ACKNOWLEDGMENT}

The author thanks Haim Brezis for bringing the Liouville question to his attention, and he thanks Yehuda Pinchover for many useful conversations and comments.

\section{REFERENCES}

[1] Arendt, W., Batty, C. J. K. and Bénilan, P. Asymptotic stability of Schrödinger semigroups on $L^{1}\left(R^{N}\right)$, Math. Z. 209 (1992), 511-518. MR1156433 (93i:47057)

[2] Batty, C. J. K. Asymptotic stability of Schrödinger semigroups: path integral methods, Math. Ann. 292 (1992), 457-492. MR1152946 (93g:47050)

[3] Ben Ari, I. and Pinsky, R. Absolute continuity/singularity and relative entropy properties for probability measures induced by diffusions on infinite time intervals, Stochastic Process. Appl. 115 (2005), 179-206. MR2111192(2005j:60145)

[4] Brezis, H., Chipot, M. and Xie, Y. preprint.

[5] Carroll, T. and Ortega-Cerda, J. Configurations of balls in Euclidean space that Brownian motion cannot avoid, Ann. Acad. Sci. Fenn. Math., to appear. MR2297888

[6] Grigor'yan, A. Bounded solutions of the Schrödinger equation on noncompact Riemannian manifolds, J. Soviet Math. 51 (1990), 2340-2349. MR1001354 (90m:35050)

[7] Grigor'yan, A. and Hansen, W. A Liouville property for Schrödinger operators, Math. Ann. 312 (1998), 659-716. MR1660247 (2000a:58092)

[8] Hess-Green, R. in preparation.

[9] John, F. Partial Differential Equations, third edition, Springer-Verlag (1978). MR514404 (80f:35001)

[10] Murata, M. Structure of positive solutions to $(-\Delta+V) u=0$ in $R^{n}$, Duke Math. J. 53 (1986), 869-943. MR874676 (88f:35039)

[11] Pinchover, Y. On the equivalence of Green functions of second order elliptic equations in $R^{n}$, Differential Integral Equations 5 (1992), 481-493. MR.1157482 (93b:35035)

[12] Pinchover, Y. Maximum and anti-maximum principles and eigenfunctions estimates via perturbation theory of positive solutions of elliptic equations, Math. Ann. 314 (1999), 555-590. MR 1704549 (2001c:35044)

[13] Pinsky, R. G. Positive Harmonic Functions and Diffusion, Cambridge University Press (1995). MR 1326606 (96m:60179)

[14] Simon, B. Schrödinger semigroups, Bull. Amer. Math. Soc. (N.S.) 7 (1982), 447-526. MR670130(86b:81001a)

Department of Mathematics, Technion-Israel Institute of Technology, Haifa, 32000, ISRAEL

E-mail address: pinsky@math.technion.ac.il 